\title{
Glucagon-like peptide-1: effect on pro-atrial natriuretic peptide in healthy males
}

\author{
Jeppe Skov ${ }^{1,2}$, Jens Juul Holst ${ }^{3}$, Jens Peter Gøtze ${ }^{4}$, Jørgen Frøkiær ${ }^{5,6}$ \\ and Jens Sandahl Christiansen ${ }^{1}$ \\ ${ }^{1}$ Department of Endocrinology and Internal Medicine, Aarhus University Hospital, Norrebrogade 44, \\ DK-8000 Aarhus, Denmark \\ ${ }^{2}$ Novo Nordisk A/S, DK-2880 Bagsvaerd, Denmark \\ ${ }^{3}$ NNF center for Basic Metabolic Research, Department of Biomedical Sciences, The Panum Institute, \\ University of Copenhagen, DK-2200 Copenhagen, Denmark \\ ${ }^{4}$ Department of Clinical Biochemistry, Rigshospitalet, University of Copenhagen, Blegdamsvej 9, \\ DK-2100 Copenhagen, Denmark \\ ${ }^{5}$ Department of Clinical Physiology and Molecular Imaging, Aarhus University Hospital, Aarhus, Denmark \\ ${ }^{6}$ Department of Clinical Medicine, Aarhus University, DK-8000 Aarhus, Denmark
}

Correspondence

should be addressed

to J Skov

Email

jsk@dadlnet.dk

\begin{abstract}
The antihypertensive actions of glucagon-like peptide-1 (GLP1) receptor agonists have been linked to the release of atrial natriuretic peptide (ANP) in mice. Whether a GLP1-ANP axis exists in humans is unknown. In this study, we examined 12 healthy young males in a randomized, controlled, double-blinded, single-day, cross-over study to evaluate the effects of a 2-h native GLP1 infusion. Plasma proANP concentrations were measured by an automated mid-region-directed proANP immunoassay and $\mathrm{N}$-terminal pro B-type natriuretic peptide (BNP) on Roche Modular E170. Urine was collected for measurements of sodium excretion. Although GLP1 infusion increased the urinary sodium excretion markedly, there were no significant changes in either proANP or proBNP concentrations. When GLP1 infusion was stopped, sodium excretion declined rapidly. As proANP concentration reflects ANP secretion, our data could not confirm the existence of a GLP1-ANP axis in humans. Especially, the natriuretic effects of GLP1 seem unlikely to be mediated exclusively via ANP.
\end{abstract}

\section{Key Words}

- glucagon-like peptide-1

- atrial natriuretic peptide

- natriuresis

- proANP

- proBNP

- kidney

- heart rate

- blood pressure

Endocrine Connections (2014) 3, 11-16

\section{Introduction}

Glucagon-like peptide-1 (GLP1) is a gut-derived incretin hormone with multiple actions in addition to control of glucose homeostasis (1). Synthetic GLP1 receptor (GLP1R) agonists lower blood pressure in patients with type 2 diabetes through mechanisms not fully comprehended (2). The antihypertensive effects seem non-acute in nature (3) but sets in before substantial weight loss (4). GLP1 has natriuretic effects in humans $(5,6)$ and some studies suggest that GLP1 has vasorelaxant effects (7).
A recently published article by Kim et al. (8) reports that atrial natriuretic peptide (ANP) mediates the antihypertensive effects of GLP1 in mice. The authors convincingly demonstrate that GLP1R activation stimulates ANP release from atrial cardiomyocytes and that GLP1 exclusively acts via ANP to induce vasodilatation and natriuresis (8). The findings, therefore, define a novel GLP1-ANP axis and expand our view on GLP1-induced cardiovascular and renal actions.

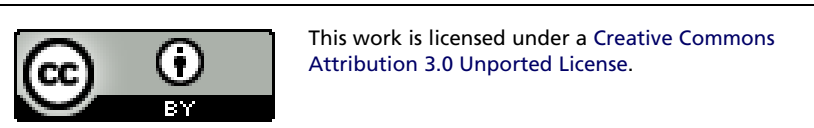


To explore whether this GLP1-ANP axis exists in humans, we measured plasma proANP concentrations in healthy males exposed to a natriuresis inducing native GLP1 infusion.

\section{Subjects and methods}

\section{Design}

The study was performed using a randomized, doubleblinded, placebo-controlled, single-day cross-over design.

\section{Subjects}

Twelve healthy male subjects were studied. Mean age was $23.4 \pm 3.1$ years (s.D.) and mean BMI was $23.0 \pm 2.0 \mathrm{~kg} / \mathrm{m}^{2}$ (s.D.). Each volunteer provided written informed consent. The protocol was approved by the local Human Ethics Research Committee and carried out in accordance with the Helsinki Declaration.

All volunteers had a normal physical examination as well as normal blood and urine screenings. None were on medication or had any history of critical medical conditions. Fourteen subjects were screened, one withdrew after screening and one was excluded after the study because of fluid retention due to impaired urinations.

\section{Protocol}

The primary study objective was to investigate GLP1induced effects on kidney function and the procedures and results regarding this part have been previously described (6). In summary, the protocol involved blood sampling, infusions of radioactive isotopes, and a prior ingestion of small doses lithium.

Volunteers arrived at the clinic at $0715 \mathrm{~h}$ after an overnight fast. They were placed in bed where they stayed supine during the study day. Catheters were placed in each antecubital vein, one for blood sampling, the other for infusion.

At $\sim 0730 \mathrm{~h}$, an isotonic $0.9 \%$ saline infusion was started at a rate of $750 \mathrm{ml} / \mathrm{h}$ and continued throughout the study to stimulate urine production. At $0900 \mathrm{~h}$ a $2-\mathrm{h}$ infusion of either GLP1, dissolved in $0.9 \%$ saline containing $1 \%$ human albumin, or the solvent alone (placebo) was initiated. This was followed by a 2-h washout period before infusion of the placebo and GLP1 solutions for the next $2 \mathrm{~h}$. The GLP1 infusion rate was $1.25 \mathrm{pmol} / \mathrm{kg}$ per min.

The two solutions were prepared by the physician in charge and afterwards blinded to both physician and volunteers allowing for the double-blinded study design. Blood samples were drawn every $20 \mathrm{~min}$, resulting in a total collection of $\sim 300 \mathrm{ml}$ blood during the entire study day. Urine was collected every $20 \mathrm{~min}$ by voiding in a bottle. Blood pressure and heart rate (HR) were measured every 20 min throughout the day.

\section{Materials}

Synthetic human GLP1(7-36) amide was obtained from Bachem (Weil am Rhein, Germany).

\section{Assays}

GLP1 plasma concentrations were measured by a RIA method as described earlier (6). For measurement of plasma proANP, we used an automated proANP immunoassay (BRAHMS, Hennigsdorf, Germany) with targeted epitopes in the mid-region (MR) of the precursor (9). Unlike ANP, MR-proANP is well preserved in plasma (10). The intra-assay coefficient of variation $(\mathrm{CV})$ from the manufacturer is $<2.5 \%$ in the relevant molar range (20$1000 \mathrm{pmol} / \mathrm{l})$; the limit of detection is $2.1 \mathrm{pmol} / \mathrm{l}$, and the limit of quantitation is listed as being $4.5 \mathrm{pmol} / \mathrm{l}$. In our laboratory, the working interassay $\mathrm{CV}$ in the current molar range was $<2 \%(n=60)$. The MR-proANP assay has also been compared with an in-house gold standard proANP assay, with excellent correlation in the low range (11).

$\mathrm{N}$-terminal pro B-type natriuretic peptide (BNP) was measured on Roche Modular E170 using a previously well-described method (12). The limit of detection was $0.6 \mathrm{pmol} / 1$ and values below this limit were set to $0.5 \mathrm{pmol} / \mathrm{l}$. Urinary sodium concentration was measured by the accredited laboratory of Aarhus University Hospital.

\section{Statistical analysis}

A mixed model of repeated measures ANOVA was applied (subject and subject $\times$ period as random variables; group, time, and group $\times$ time as fixed variables). The $P$ value of the interaction group $\times$ time is reported throughout. The proBNP data were logarithmically transformed before analyses. A $P<0.05$ is considered statistically significant. STATA, version 11.1 (StataCorp., College Station, TX, USA) was used for calculations.

\section{Results}

When infusion of native GLP1 $1.25 \mathrm{pmol} / \mathrm{kg}$ per min started, the baseline GLP1 concentration increased nearly

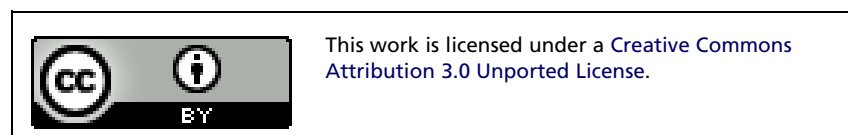


tenfold and remained elevated until the infusion was terminated $(P<0.001)$. The urinary sodium excretion increased $\sim 40 \%(P<0.001)$ during GLP1 infusion but rapidly returned toward baseline when infusion stopped (as shown for the group randomized to GLP1 in the first intervention period, $n=6$ ). GLP1 infusion did not affect proANP plasma concentration $(P=0.32$; see Fig. 1$)$. Both proANP and proBNP concentrations increased slightly over time independent of the order of GLP1 and placebo infusions.

There was no acute impact on either systolic blood pressure (SBP; $P=0.31$ ) or diastolic blood pressure (DBP; $P=0.63)$, but HR transiently increased by approximately six beats per minute $(P=0.01)$. The effect on vital parameters has previously been published (6) (see Fig. 2).

\section{Discussion}

We have shown that an almost tenfold increase in GLP1 concentration, which markedly induced natriuresis, was incapable of increasing plasma proANP or proBNP concentrations in healthy males. As proANP concentration reflects ANP secretion, the result questions the existence of a functional GLP1-ANP axis in humans. Especially the natriuretic effect seems unlikely to be mediated exclusively via ANP.

\section{GLP1 does not increase proANP concentration}

Despite a robust and sustained increase in GLP1 concentrations during the 2-h GLP1 intervention, we were unable to detect an increase in proANP plasma concentration.
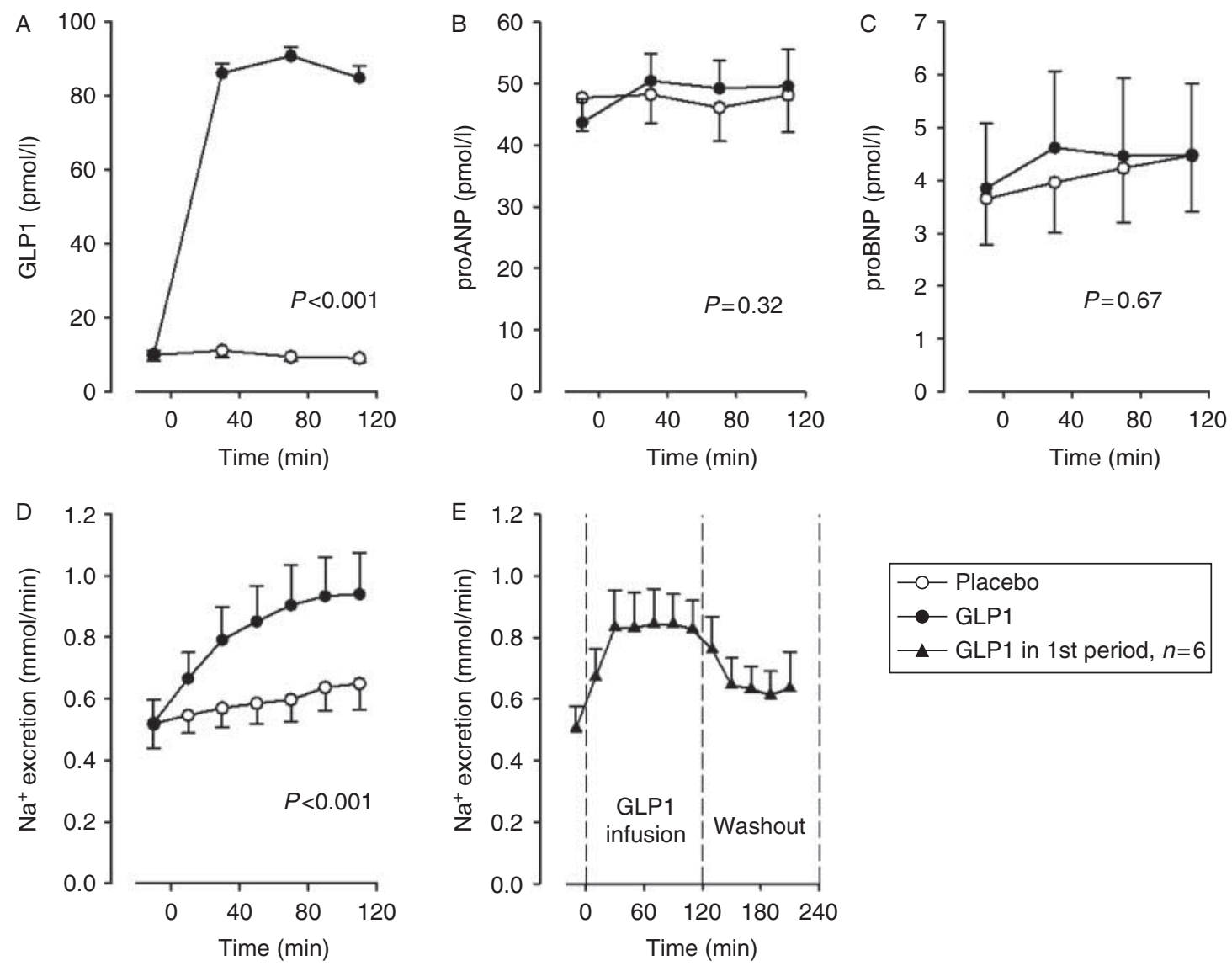

\section{Figure 1}

GLP1 infusion increased plasma GLP1 concentration nearly tenfold, (A) but this had no significant effect on plasma proANP (B) or proBNP (C) concentrations despite a marked increase in urinary sodium excretion (D). Sodium excretion rapidly declined when GLP1 infusion stopped (E). Open circles represent values from the placebo period; closed circles

\begin{tabular}{|lr} 
http://www.endocrineconnections.org & @ 2014 The authors \\
DOI: $10.1530 /$ EC-13-0087 & Published by Bioscientifica Ltd
\end{tabular}

from the GLP1 period; and triangles represent data from subjects randomized to GLP1 in the first intervention period $(n=6)$. Statistical significance was determined by ANOVA. Data are shown as mean and error bars represent S.E.M. 

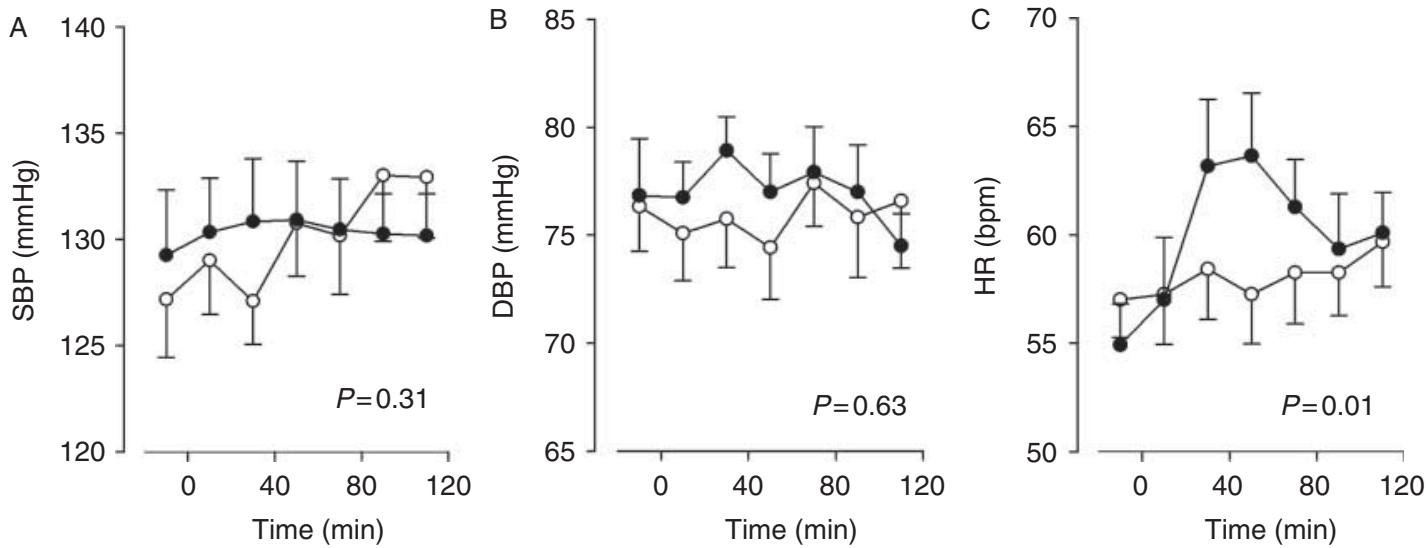

\section{Figure 2}

GLP1 infusion had no acute effect on either SBP (A) or DBP (B) but there was a transient increase in heart rate (C). Open circles represent values from the placebo period and closed circles from the GLP1 period.

Plasma proANP is today used clinically as a surrogate marker for cardiac ANP secretion. The prohormone has a longer half-life in the circulation, shows smaller fluctuations, and the peptide is better preserved in plasma samples (10).

The half-life of ANP is $<5 \mathrm{~min}$, and while the exact half-life of proANP is unknown, we have seen $50 \%$ reduction of the proANP concentration in vivo within 60 min (13). This should facilitate our ability to detect a rise in the secretion of ANP (with its synchronous and equimolar secretion of proANP).

In the article by Kim et al. (8), the majority of their numerous experiments were performed with the GLP1R agonist liraglutide. However, by using GLP1R knockout mice, they showed that ANP release indeed depended on GLP1R activations; in addition, exendin-4, native GLP1, as well as a meal (endogenous GLP1) were capable of increasing ANP concentrations.

We would therefore expect native GLP1 to stimulate ANP secretion in humans if the same mechanisms were present. As GLP1 failed to increase proANP levels, we conclude that either the GLP1-ANP axis is solely a mouse phenomenon or that a GLP1 induced release of ANP in humans must be minor or very transient.

A recent paper exploring the effect of a meal on plasma proANP concentrations in humans (13) also speaks against a major effect of GLP1 on ANP secretion. Six overnight fasted healthy young individuals were given a standardized meal and proANP was measured repeatedly for the first three postprandial hours. The meal was found to
Statistical significance was determined by ANOVA. Data are shown as mean and error bars represent S.E.M.

significantly reduce proANP concentrations. Since a meal is the physiological stimulus for GLP1 secretion we would expect the meal to increase proANP if a GLP1-ANP axis existed in humans.

\section{No effect of GLP1 on proBNP}

We also measured N-terminal-proBNP as a marker of BNP secretion, but found no effect in response to GLP1 infusion. Kim et al. (8) did not either find BNP to be a mediator of GLP1-actions in mice. It seems therefore reasonable to conclude that $\mathrm{BNP}$ is not part of an important gut-heart axis.

\section{ANP is not exclusively mediating GLP1's natriuretic actions}

We found infusion of native GLP1 to increase the urinary sodium excretion markedly in humans (Fig. 1D). Further, the increase in natriuresis was sustained during the entire period of GLP1 infusion, but rapidly returned toward baseline when infusion was stopped (Fig. 1E). As there were no changes in proANP concentrations, the finding is inconsistent with ANP being an essential mediator of GLP1's natriuretic actions in humans.

Kim et al. (8) found liraglutide to markedly increase natriuresis in mice and thereby confirmed an earlier finding of liraglutide-induced diuresis in rats (14). Liraglutide had no effect on natriuresis in ANP knockout mice, indicating that ANP is required for the natriuretic actions of GLP1R agonists (8). The different GLP1R 
agonists often induced an initial peak in ANP level, which disappeared within the first hour (8). This was especially the case during the light-on periods. We found the increased urinary sodium excretion to be sustained under the GLP1 infusion and hereafter to decrease rapidly. Therefore, it is unlikely that the effect was caused by a hypothetical initial ANP peak not reflected by increases in proANP concentrations.

There may, however, be important differences between native GLP1 and liraglutide with respect to renal actions. The natriuretic effect has been hypothesized to depend on activation of GLP1Rs located in the proximal tubules (15). While GLP1 is freely filtered and partly cleared in the kidneys, only minimal amounts of liraglutide pass the glomerular filtration barrier (16). If GLP1R activation depends on GLP1R agonists located in the tubular lumen, liraglutide may not be functioning. Though liraglutide induces natriuresis in rodents $(8,14)$, to our knowledge, it has never been reported whether liraglutide actually has natriuretic properties in humans.

ANP infusion markedly increases glomerular filtration rate in rodents $(17,18)$ and humans $(19,20)$. GLP1 infusion also strikingly increases glomerular filtration rate in rodents $(15,21)$ but interestingly not in humans $(5,6)$. This difference could be explained by a GLP1-ANP axis which is only functional in rodents.

\section{No acute effect of GLP1 on blood pressure}

We did not find native GLP1 infusion to acutely affect blood pressure in healthy males (Fig. 2), which we have also prior reported (6). Kim et al. (8) found different GLP1R agonists to reduce blood pressure but only in mice which were hypertensive secondary to an angiotensin II infusion or to transaortic constriction, and the effects were most pronounced during the light-off periods. Our findings, therefore, are not in contrast, because the volunteers were normotensive. Further, the results are also in line with other human studies showing no acute effect of GLP1R agonists on blood pressure (3). A GLP1-induced increase in HR (Fig. 2C) has been reported in both rodents and humans, although usually not reported to be transient. Notably, Kim et al. (8) found that the positive chronotropic effect of GLP1R agonists was independent of ANP.

\section{Implications for proANP as a biomarker of heart failure}

Mid-regional proANP has been introduced as a heart failure biomarker (22). Kim et al. (8) found GLP1R agonists to markedly increase ANP levels in mice but found no (c) 2014 The authors Published by Bioscientifica Ltd effect on BNP. This indirectly raises the question whether proANP is a valid biomarker of heart failure in patients treated with GLP1-based products or if derives of BNP are better biomarkers in these cases. Diabetes per se is known to increase the risk of heart failure, and rising numbers of patients are now treated with GLP1 products which make the concern for false positive results highly relevant.

Our data showed no impact on proANP or proBNP after GLP1 infusion. Consequently, we have no reason to believe that either proANP or proBNP are inadequate biomarkers of heart failure in patients treated with GLP1based products. The question, however, demands further investigations in type 2 diabetes patients treated with GLP1R agonists for longer periods.

The major limitation of this study is that it was not originally designed to evaluate a GLP1-ANP axis and the proANP analyses were performed post-hoc. Further, we did not measure ANP itself because our blood samples were not properly pretreated to allow for acceptable analyses of this highly unstable peptide. High saline infusion rates and frequent blood sampling may have induced period effects.

In conclusion, our data cannot confirm the existence of a GLP1-ANP axis in humans and we find especially GLP1's natriuretic effect unlikely to be caused exclusively via increased ANP secretion. The topic is important because increasing numbers of patients are treated with GLP1-based products which make cardiovascular side effects an obvious topic of interest. Dedicated studies are needed to further explore the GLP1-ANP relationship in humans.

\section{Declaration of interest}

J J Holst, J Frøkiær, and J P Goetze have nothing to declare in relation to this study. J Skov is employed by Novo Nordisk in a PhD fellowship. J S Christiansen is a recipient of unrestricted research grants from Novo Nordisk, is a clinical investigator for Novo Nordisk, receives lecture fees from Novo Nordisk, and is a member of Novo Nordisk advisory boards.

\section{Funding}

This work was supported by Novo Nordisk A/S, the Danish Agency for Science Technology and Innovation, and Aarhus University Hospital.

\section{References}

1 Ussher JR \& Drucker DJ. Cardiovascular biology of the incretin system. Endocrine Reviews 201233 187-215. (doi:10.1210/er.2011-1052)

2 Robinson LE, Holt TA, Rees K, Randeva HS \& O'Hare JP. Effects of exenatide and liraglutide on heart rate, blood pressure and body weight: systematic review and meta-analysis. BMJ Open $20133 \mathrm{e} 001986$. (doi:10.1136/bmjopen-2012-001986) http://www.endocrineconnections.org DOI: 10.1530/EC-13-0087

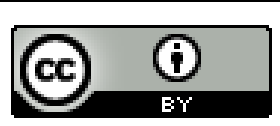

This work is licensed under a Creative Commons Attribution 3.0 Unported License. 
3 Mendis B, Simpson E, Macdonald I \& Mansell P. Investigation of the haemodynamic effects of exenatide in healthy male subjects. British Journal of Clinical Pharmacology 201274 437-444. (doi:10.1111/ j.1365-2125.2012.04214.x)

4 Varanasi A, Chaudhuri A, Dhindsa S, Arora A, Lohano T, Vora MR \& Dandona P. Durability of effects of exenatide treatment on glycemic control, body weight, systolic blood pressure, C-reactive protein, and triglyceride concentrations. Endocrine Practice 201117 192-200. (doi:10.4158/EP10199.OR)

5 Gutzwiller JP, Tschopp S, Bock A, Zehnder CE, Huber AR, Kreyenbuehl M, Gutmann H, Drewe J, Henzen C, Goeke B et al. Glucagon-like peptide 1 induces natriuresis in healthy subjects and in insulin-resistant obese men. Journal of Clinical Endocrinology and Metabolism 200489 3055-3061. (doi:10.1210/jc.2003-031403)

6 Skov J, Dejgaard A, Frokiaer J, Holst JJ, Jonassen T, Rittig S \& Christiansen JS. Glucagon-like peptide-1 (GLP-1): effect on kidney hemodynamics and renin-angiotensin-aldosterone system in healthy men. Journal of Clinical Endocrinology and Metabolism 201398 E664-E671. (doi:10.1210/jc.2012-3855)

7 Nystrom T, Gutniak MK, Zhang Q, Zhang F, Holst JJ, Ahren B \& Sjoholm A. Effects of glucagon-like peptide- 1 on endothelial function in type 2 diabetes patients with stable coronary artery disease. American Journal of Physiology. Endocrinology and Metabolism 2004287 E1209-E1215. (doi:10.1152/ajpendo.00237.2004)

8 Kim M, Platt MJ, Shibasaki T, Quaggin SE, Backx PH, Seino S, Simpson JA \& Drucker DJ. GLP-1 receptor activation and Epac2 link atrial natriuretic peptide secretion to control of blood pressure. Nature Medicine 201319 567-575. (doi:10.1038/nm.3128)

9 Morgenthaler NG, Struck J, Thomas B \& Bergmann A. Immunoluminometric assay for the midregion of pro-atrial natriuretic peptide in human plasma. Clinical Chemistry 200450 234-236. (doi:10.1373/clinchem.2003.021204)

10 Buckley MG, Marcus NJ \& Yacoub MH. Cardiac peptide stability, aprotinin and room temperature: importance for assessing cardiac function in clinical practice. Clinical Science 199997 689-695. (doi:10.1042/CS19990194)

11 Hunter I, Alehagen U, Dahlstrom U, Rehfeld JF, Crimmins DL \& Goetze JP. N-terminal pro-atrial natriuretic peptide measurement in plasma suggests covalent modification. Clinical Chemistry 201157 1327-1330. (doi:10.1373/clinchem.2011.166330)
12 Goetze JP, Dahlstrom U, Rehfeld JF \& Alehagen U. Impact of epitope specificity and precursor maturation in pro-B-type natriuretic peptide measurement. Clinical Chemistry 200854 1780-1787. (doi:10.1373/ clinchem.2008.105635)

13 Goetze JP. Plasma proANP decreases after meal intake. Clinical Chemistry 201359 1270-1271. (doi:10.1373/clinchem.2012.202416)

14 Larsen PJ, Fledelius C, Knudsen LB \& Tang-Christensen M. Systemic administration of the long-acting GLP-1 derivative NN2211 induces lasting and reversible weight loss in both normal and obese rats. Diabetes 200150 2530-2539. (doi:10.2337/diabetes.50.11.2530)

15 Crajoinas RO, Oricchio FT, Pessoa TD, Pacheco BP, Lessa LM, Malnic G $\&$ Girardi AC. Mechanisms mediating the diuretic and natriuretic actions of the incretin hormone glucagon-like peptide-1. American Journal of Physiology. Renal Physiology 2011301 F355-FF63. (doi:10.1152/ajprenal.00729.2010)

16 Malm-Erjefalt M, Bjornsdottir I, Vanggaard J, Helleberg H, Larsen U, Oosterhuis B, van Lier JJ, Zdravkovic M \& Olsen AK. Metabolism and excretion of the once-daily human glucagon-like peptide- 1 analog liraglutide in healthy male subjects and its in vitro degradation by dipeptidyl peptidase IV and neutral endopeptidase. Drug Metabolism and Disposition 201038 1944-1953. (doi:10.1124/dmd.110.034066)

17 Beasley D \& Malvin RL. Atrial extracts increase glomerular filtration rate in vivo. American Journal of Physiology 1985248 F24-F30.

18 Cogan MG. Atrial natriuretic factor can increase renal solute excretion primarily by raising glomerular filtration. American Journal of Physiology 1986250 F710-F714

19 Weidmann P, Hasler L, Gnadinger MP, Lang RE, Uehlinger DE, Shaw S, Rascher W \& Reubi FC. Blood levels and renal effects of atrial natriuretic peptide in normal man. Journal of Clinical Investigation 198677 734-742. (doi:10.1172/JCI112368)

20 Cuocolo A, Volpe M, Mele AF, Celentano L, Neumann RD, Trimarco B \& Salvatore M. Effects of atrial natriuretic peptide on glomerular filtration rate in essential hypertension: a radionuclide study. European Journal of Nuclear Medicine 199118 32-37. (doi:10.1007/ BF00177682)

21 Moreno C, Mistry M \& Roman RJ. Renal effects of glucagon-like peptide in rats. European Journal of Pharmacology 2002434 163-167. (doi:10.1016/S0014-2999(01)01542-4)

22 Gaggin HK \& Januzzi JL Jr. Biomarkers and diagnostics in heart failure. Biochimica et Biophysica Acta 20131832 2442-2450. (doi:10.1016/ j.bbadis.2012.12.014)

Received in final form 10 November 2013

Accepted 5 December 2013 http://www.endocrineconnections.org

DOI: 10.1530/EC-13-0087
(C) 2014 The authors Published by Bioscientifica Ltd

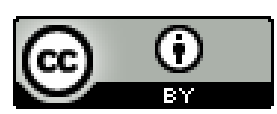

This work is licensed under a Creative Commons Attribution 3.0 Unported License. 